\title{
Number of Siblings
}

National Cancer Institute

\section{Source}

National Cancer Institute. Number of Siblings. NCI Thesaurus. Code C102469.

An individual's total number of brothers and sisters. 